\section{Laguerre-Gram reduced-order modeling}

Ahmed Amghayrir, Student Member, IEEE, Noël Tanguy, Pascale Bréhonnet, Pierre Vilbé, and Léon-Claude Calvez

\begin{abstract}
We present an efficient model reduction procedure based on the Laguerre description of the system to be approximated. Using a one-order operator defined in the Laplace domain we construct a pencil of functions and formulate the problem as the minimization of the $L_{\varpi}^{2}\left(\mathbb{R}^{+}\right)$criterion. The use of a weight function in the inner product definition allows a control of the time-error spreading in model reduction procedure. We show how the required Gram matrix can be computed efficiently and prove that the impulse response of the reduced model is also in $L_{\varpi}^{2}\left(\mathbb{R}^{+}\right)$. The transfer function approach allows an immediate and promising application in model reduction of infinite dimensional systems. An extension to MIMO systems is also given.
\end{abstract}

Index Terms-Model-order reduction, Laguerre functions, infinite dimensional systems.

\author{
Corresponding address: \\ Noël TANGUY \\ LEST, UMR CNRS n ${ }^{\circ} 6165$ \\ UFR Sciences et Techniques, C.S. 93837 \\ 6 avenue Le Gorgeu \\ 29238 BREST CEDEX 3, FRANCE \\ Tel: (33) 2.98.01.61.26 \\ Fax: (33) 2.98.01.63.95 \\ e-mail: Noel.Tanguy@univ-brest.fr
}

\section{INTRODUCTION}

Laguerre functions have shown their large potential in numerous applications as in signal analysis and parameter identification [1], system identification [2], [3], approximation of finite or infinitedimensional system [4], [5], industrial control [6],... More recently, starting with a state-space representation of a system, a Laguerre description has been used to compute a reduced-order model of the system. The method is based on Padé approximation of the Laguerre spectra, associated to a Krylov subspace decomposition technique [7]-[9]. In this paper we present a model reduction technique using a transfer-function formalism that allows reduction of irrational transfer functions. The derivative and integral operators usually employed to construct a pencil of functions for the reduction procedure are here replaced by a one-order operator more suited to construct a set of basis functions from the Laguerre description of the system. The model reduction problem is then formulated as a $L_{\varpi}^{2}\left(\mathbb{R}^{+}\right)$ criterion minimization in which the required Gram matrix can be efficiently computed. The use of a weight function in the inner product definition permits a control of the time-error spreading of the reduced model. Assuming that the original function belongs to $L_{\varpi}^{2}\left(\mathbb{R}^{+}\right)$the presented procedure yields a reduced model whose impulse response provably belongs to $L_{\varpi}^{2}\left(\mathbb{R}^{+}\right)$. An extension to MIMO systems is also derived.

The paper is organized as follows. From the Laguerre representation of the system we construct in section II a set of linearly independent functions. On the base of this set of functions we formulate in section III the model reduction problem as the minimization of a $L_{\varpi}^{2}\left(\mathbb{R}^{+}\right)$criterion and solve it. The belonging to $L_{\varpi}^{2}\left(\mathbb{R}^{+}\right)$of the resultant funtion is proved and the extension of the reduction procedure to MIMO systems is given. A numerical example of the model reduction method is then presented in section IV.

The authors are with the Laboratoire d'Electronique et des Systèmes de Télécommunications (LEST) UMR CNRS n ${ }^{\circ} 6165$ at the University of Brest, Brest, France.

\section{Pencil of FUnCtions CONSTRUCTION}

The real causal two-parameters Laguerre functions $\phi_{n}(t)$ related to the Laguerre polynomials $L_{n}(x)$ by

$$
\phi_{n}(t) \triangleq \sqrt{\gamma} e^{-\alpha t} L_{n}(\gamma t),
$$

with $\alpha>0, \gamma>0$ and

$$
L_{n}(x) \triangleq \frac{e^{x}}{n !} \frac{d^{n}}{d x^{n}}\left(x^{n} e^{-x}\right),
$$

have the following Laplace transforms

$$
\hat{\phi}_{n}(s)=\frac{\sqrt{\gamma}}{s+\alpha}\left(\frac{s-\gamma+\alpha}{s+\alpha}\right)^{n}, \quad n=0,1,2, \ldots
$$

Defining the weighted inner product of two real causal functions by

$$
\langle f, g\rangle \triangleq \int_{0}^{\infty} \varpi(t) f(t) g(t) d t,
$$

where the weight function is given by

$$
\varpi(t) \triangleq e^{-(\gamma-2 \alpha) t},
$$

let $L_{\varpi}^{2}\left(\mathbb{R}^{+}\right)$denote the Hilbert space of measurable $\mathbb{R}$-valued functions $f(t)$ for which $\langle f, f\rangle \triangleq\|f\|_{\varpi}^{2}<\infty$. It will be noted that a necessary (but not sufficient) condition for $f(t)$ to belong to $L_{\varpi}^{2}\left(\mathbb{R}^{+}\right)$is $\frac{\gamma}{2}-\alpha>\Re\left(-s_{i}\right)$, where $\left(-s_{i}\right)$ stands for the poles of $\hat{f}(s)$ [10]. The Laguerre functions constitute an orthonormal basis in $L_{\varpi}^{2}\left(\mathbb{R}^{+}\right)$satisfying

$$
\left\langle\phi_{n}, \phi_{m}\right\rangle=\delta_{n, m}
$$

with $\delta_{n, m}$ the Krönecker symbol. In mostly engineering literature the particular choice $\gamma-2 \alpha=0$ is made and corresponds to an unit weight function in the inner product definition (2). A different choice of the $(\gamma-2 \alpha)$ value modifies the temporal part of the functions in the inner product and will yield to modify the error-spreading of the impulse response of the reduced model along the temporal axis. Since the Laguerre functions constitute an orthonormal basis in $L_{\varpi}^{2}\left(\mathbb{R}^{+}\right)$, the Laplace transform $\hat{f}(s)$ of any function $f \in L_{\varpi}^{2}\left(\mathbb{R}^{+}\right)$can be represented by a Laguerre series

$$
\hat{f}(s)=\sum_{n=0}^{\infty} f_{n} \hat{\phi}_{n}(s),
$$

where the Laguerre spectrum $\left\{f_{n}\right\}_{n \geq 0}$ is given by $f_{n}=\left\langle f, \phi_{n}\right\rangle$. Using the orthogonality property, the weighted inner product defined in (2) can be replaced by a discrete sum usually more convenient for numerical computation

$$
\langle f, g\rangle=\sum_{n=0}^{\infty} f_{n} g_{n}
$$

Denoting $F(z)$ the $z$-transform of the Laguerre spectrum $\left\{f_{n}\right\}_{n>0}, F(z)$ can be derived using the bilinear transformation $z=\frac{s+\alpha}{s+\alpha-\gamma}$ which maps the open left plane in $s$-domain delimited by $\Re(s)<\frac{\gamma}{2}-\alpha$ into the open unit disk in $z$-domain

$$
\hat{f}(s) \mapsto F(z) \triangleq \sum_{n=0}^{\infty} f_{n} z^{-n}=\frac{\sqrt{\gamma} z}{z-1} \hat{f}\left(\frac{\gamma z}{z-1}-\alpha\right) .
$$

It is worth noting that the inner product (4) may be written in the $z$-domain as

$$
\langle f, g\rangle=\frac{1}{2 \pi j} \oint_{|z|=1} F(z) G\left(\frac{1}{z}\right) \frac{d z}{z} .
$$

An important property of the bilinear transformation is the preservation of the order of complexity of transfer functions. As for example if $\hat{f}(s)$, an irreducible analytic transfer function in some 
$s$-domain, is given by $\hat{f}(s)=\frac{p_{m-1} s^{m-1}+\ldots+p_{1} s+p_{0}}{q_{m} s^{m}+\ldots+q_{1} s+q_{0}}$ therefore $F(z)=\frac{z\left(u_{m-1} z^{m-1}+\ldots+u_{1} z+u_{0}\right)}{v_{m} z^{m}+\ldots+v_{1} z+v_{0}}$ will be an irreducible, analytic and rational transfer function in corresponding $z$-domain. The reciprocal transformation giving $\hat{f}(s)$ from $F(z)$ is

$$
F(z) \mapsto \hat{f}(s)=\frac{\sqrt{\gamma}}{s+\alpha} F\left(\frac{s+\alpha}{s-\gamma+\alpha}\right) .
$$

Now let us define

$$
\hat{f}(s) \mapsto \Lambda\{\hat{f}(s)\} \triangleq \frac{s+\alpha}{s-\gamma+\alpha}\left[\hat{f}(s)-\frac{\gamma}{s+\alpha} \hat{f}(\gamma-\alpha)\right]
$$

and construct recursively $\Omega_{r}=\left\{\hat{f}_{0}, \hat{f}_{1}, \ldots, \hat{f}_{r}\right\}$ using

$$
\begin{aligned}
\hat{f}_{0}(s) & \triangleq \hat{f}(s), \\
\hat{f}_{i+1}(s) & \triangleq \Lambda\left\{\hat{f}_{i}(s)\right\}
\end{aligned}
$$

for $i=0,1, \ldots, r-1$. Denoting $\left\{f_{i, n}\right\}_{n \geq 0}$ the Laguerre spectra of the functions $\hat{f}_{i}(s)$ and $\Xi_{r}=\left\{F_{0}, F_{1}, \ldots, F_{r}\right\}$ the set of functions $F_{i}(z)$ derived from $\hat{f}_{i}(s)$ via transformation (5), one obtains the relation linking the successive functions $F_{i}(z)$ as

$$
F_{i+1}(z)=z\left[F_{i}(z)-f_{i, 0}\right]
$$

with $F_{0}(z)=F(z)$. Therefore it follows that the Laguerre spectrum of $\hat{f}_{i+1}(s)$ is readily obtained from the Laguerre spectrum of $\hat{f}_{i}(s)$, and so on

$$
f_{i+1, n}=f_{i, n+1}=\ldots=f_{0, n+i+1}=f_{n+i+1}
$$

for all $n \geq 0, i \geq 0$. Hence no computation is required and so no error is made when computing the Laguerre spectrum of any member of the set $\Omega_{r}$. Moreover using (8) or (9) and $f_{i, 0}=\sqrt{\gamma} \hat{f}_{i}(\gamma-\alpha)=$ $\lim _{z \rightarrow \infty} F_{i}(z)$ deduced from (5) one should readily verify that $\Lambda$ operator preserves the natural frequencies or poles in the Laplace domain. Therefore $\Omega_{r}$ constitutes an efficient set of approximating functions for determining a $r$-order reduced denominator.

\section{MODEL REDUCTION}

To construct a $r$-order reduced model of $\hat{f}(s)$, consider now the approximation of $\hat{f}_{r}(s)$ using the set $\Omega_{r-1}$. Denoting $\hat{e}(s)$ the local error and $a_{i}$ the negatives of the coefficients of the approximation, we can write

$$
\sum_{i=0}^{r} a_{i} \hat{f}_{i}(s)+\hat{e}(s)=0, \quad \text { where } a_{r}=1 .
$$

Applying transformation (5) to equation (11) yields

$$
\sum_{i=0}^{r} a_{i} F_{i}(z)+E(z)=0,
$$

where

$$
E(z)=\frac{\sqrt{\gamma} z}{z-1} \hat{e}\left(\frac{\gamma z}{z-1}-\alpha\right)
$$

Therefore, in view of (6), we infer that minimizing $\langle e, e\rangle$ in Laplace domain to construct an $r$-order reduced model of $\hat{f}(s)$ is equivalent to minimizing $\langle e, e\rangle$ in $z$-domain to construct an $r$-order reduced model of $F(z)$.

Denoting $\Psi$ the Gram matrix constituted of the inner products $\psi_{i, j} \triangleq\left\langle f_{i}, f_{j}\right\rangle$ for $i, j=0,1, \ldots, r-1$ and $\vec{b} \triangleq$ $\left[\begin{array}{llll}\psi_{0, r} & \psi_{1, r} & \ldots & \psi_{r-1, r}\end{array}\right]^{T}$ where ${ }^{T}$ denotes the transpose, then the best $r$-vector $\vec{a} \triangleq\left[\begin{array}{llll}a_{0} & a_{1} & \ldots & a_{r-1}\end{array}\right]^{T}$ in the sense of minimizing the error energy $\langle e, e\rangle$ in (11) can be obtained by solving the linear system

$$
\Psi \vec{a}=-\vec{b}
$$

From (4) and (10) the inner products can be expressed exclusively from the Laguerre spectrum of $\hat{f}(s)$ as

$$
\psi_{i, j}=\sum_{n=0}^{\infty} f_{n+i} f_{n+j},
$$

which constitute absolutely convergent series assuming that $f(t)$ belongs to $L_{\varpi}^{2}\left(\mathbb{R}^{+}\right)$. Inner products $\psi_{i, j}$ are thus replaced by sums, in practice finite, that are simple to calculate. Moreover, cost computation of the Gram matrix will be drastically reduced using the symmetry property $\psi_{i, j}=\psi_{j, i}$ and the recurrence relation deduced from (14)

$$
\psi_{i, j}=\psi_{i-1, j-1}-f_{i-1} f_{j-1}, \quad i, j=1,2, \ldots
$$

Repeating the use of (9) and using (10) yields

$$
F_{i}(z)=z^{i} F_{0}(z)-\sum_{j=0}^{i-1} f_{j} z^{i-j} .
$$

Substituting (16) in (12) and solving for $F_{0}(z)$ yields

$$
F_{0}(z)=\frac{z B_{r-1}(z)-E(z)}{A_{r}(z)}
$$

where $B_{r-1}(z) \triangleq \sum_{i=1}^{r} a_{i} \sum_{j=0}^{i-1} f_{j} z^{i-j-1}$ and $A_{r}(z) \triangleq \sum_{i=0}^{r} a_{i} z^{i}$, with $a_{r}=1$, are polynomials in $z$ respectively of order $r-1$ and $r$. Because $E(z)$ is an error term, assuming that $\langle e, e\rangle$ is sufficiently small, equation (17) suggests that

$$
\tilde{F}(z)=\frac{z B_{r-1}(z)}{A_{r}(z)}
$$

is a $r$-order candidate model for $F(z)=F_{0}(z)$. Therefore applying reciprocal transformation (7) to (18) yields

$$
\widehat{\widetilde{f}}(s)=\frac{p(s)}{q(s)}=\frac{\sqrt{\gamma} \sum_{i=1}^{r} a_{i} \sum_{j=0}^{i-1} f_{j}(s+\alpha)^{i-j-1}(s-\gamma+\alpha)^{r-i+j}}{\sum_{i=0}^{r} a_{i}(s+\alpha)^{i}(s-\gamma+\alpha)^{r-i}}
$$

with $a_{r}=1$, a $r$-model candidate for approximating $\hat{f}(s)$.

Starting with the Laguerre spectrum $\left\{f_{n}\right\}_{n \geq 0}$ of $\hat{f}(s)$, the model reduction procedure to construct a $r$-order model of $\hat{f}(s)$ can be summarized as follows:

1. Form the Gram matrix $\Psi$ and $\vec{b} \triangleq\left[\psi_{0, r} \psi_{1, r} \ldots \psi_{r-1, r}\right]^{T}$, by computing the required inner products in the following way:

- compute $\psi_{0, j}=\sum_{n=0}^{\infty} f_{n} f_{n+j}$ for $j=0,1, \ldots r$,

- recursively compute $\psi_{i, j}=\psi_{i-1, j-1}-f_{i-1} f_{j-1}$, for $i=$ $1,2, \ldots r-1$ and $j=i, i+1, \ldots, r$,

- complete the lower triangular part of $\Psi$ using the symmetry property $\psi_{i, j}=\psi_{j, i}$.

2. Solve $\Psi \vec{a}=-\vec{b}$ for $\vec{a}=\left[\begin{array}{llll}a_{0} & a_{1} & \ldots & a_{r-1}\end{array}\right]^{T}$.

3. Form the reduced model using (19).

Remarks:

- The Laguerre expansion for some simple transfer functions can be found in [11], but usually deducing the infinite Laguerre expansion (3) and computing the Gram matrix $\Psi$ of an irrational transfer function is not a straightforward problem. In practice a $N$-order truncated Laguerre series $(N \gg r)$ should be used in the place of (3); assuming that the truncated error is sufficiently small the model reduction will give a good approximation of the original transfer function. It will be noted that the Laguerre spectrum $\left\{f_{n}\right\}_{0 \leq n<N}$ of $\hat{f}(s)$, can always be evaluated by the Weeks method [12], [13]. 
- It will be noted that the reduced model given by (19) preserves the $r$-first Laguerre coefficients $f_{j}$ of $\hat{f}(s)$. As the Laguerre spectrum of $\hat{f}(s)$ is related to the derivatives of $\hat{f}(s)$ at $s=\gamma-\alpha$ [11] by

$$
\left.\frac{d^{n} \hat{f}(s)}{d s^{n}}\right|_{s=\gamma-\alpha}=\frac{1}{\sqrt{\gamma}} \frac{n !}{(-\gamma)^{n}} \sum_{j=0}^{n}\left(\begin{array}{l}
n \\
j
\end{array}\right)(-1)^{j} f_{j}
$$

where $\left(\begin{array}{c}n \\ j\end{array}\right)$ stands for the binomial coefficients, this implies that the reduced model given by (19) preserves the first derivatives of $\hat{f}(s)$ at $s=\gamma-\alpha$, i.e.

$$
\left.\frac{d^{n} \widehat{\widetilde{f}}(s)}{d s^{n}}\right|_{s=\gamma-\alpha}=\left.\frac{d^{n} \hat{f}(s)}{d s^{n}}\right|_{s=\gamma-\alpha}, \quad n=0,1, \ldots, r-1 .
$$

- Moreover, taking $q(s)=\sum_{i=0}^{r} a_{i}(s+\alpha)^{i}(s-\gamma+\alpha)^{r-i}$ as the denominator of the reduced model, the choice of the numerator can be optimized in the sense of minimizing the quadratic error $\langle f-\tilde{f}, f-\tilde{f}\rangle$. The problem is then a well-known linear problem which leads to the resolution of $\widehat{\widetilde{f}}\left(\nu_{i}^{*}+\gamma-2 \alpha\right)=\hat{f}\left(\nu_{i}^{*}+\gamma-2 \alpha\right)$ for $i=1,2, \ldots, r$ where $\nu_{i}^{*}$ stands for the negatives of the complex conjugate of the zeros of $q(s)$.

\section{A. Belonging to $L_{\varpi}^{2}\left(\mathbb{R}^{+}\right)$consideration}

Assuming that $\hat{f}(s)$ is a transfer function of degree $r_{0} \geq r, \Omega_{r-1}$ is a set of linearly independent functions and then the Gram matrix $\Psi$ constituted of the inner products $\psi_{i, j}=\left\langle f_{i}, f_{j}\right\rangle(i, j=0,1, \ldots, r-1)$ is a positive definite matrix. Consider now the Lyapunov equation $\Psi-$ $A^{T} \Psi A=C$ where $A \triangleq\left(\begin{array}{c|c}\overrightarrow{0}^{T} & -\vec{a} \\ I & \end{array}\right)$ represents the companion form of the reduced system in the state-space representation. Taking into account that $\vec{a}$ satisfies (13) and using (15), the solution of the Lyapunov equation is given by $C=\overrightarrow{c c}^{T}+M$ where $\vec{c} \triangleq$ $\left[\begin{array}{llll}f_{0} & f_{1} & \ldots & f_{r-1}\end{array}\right]^{T}$ and $M \triangleq\left(\begin{array}{c|c}0 & \overrightarrow{0} \\ \overrightarrow{0}^{T} & \varepsilon\end{array}\right)$ with $\varepsilon=\langle e, e\rangle$. As the quadratic error $\varepsilon$ is always non-negative, $C$ matrix is semi-positive definite, therefore $A_{r}(z)$ cannot have any zero outside the unit circle and thus $q(s)$ cannot have any zero in the right half-plane delimited by $\Re(s)>\frac{\gamma}{2}-\alpha$. Furthermore, provided that $\{A, C\}$ is observable, the reduced model belongs to $L_{\varpi}^{2}\left(\mathbb{R}^{+}\right)$[14]. In the particular case $\gamma=2 \alpha$, which corresponds to a unit weighting function in (2), the model-reduction procedure then yields a model proved to be asymptotically stable.

\section{B. Extension to MIMO systems}

In MIMO case, the system under consideration is described by a matrix of transfer functions $\widehat{\mathbf{F}}(s)=\left[\widehat{f}_{\lambda, \mu}(s)\right]$. The problem is then to derive a matrix $\widehat{\widetilde{\mathbf{F}}}(s)$ of transfer functions possessing the same $r$-order denominator $q(s)$ i.e.

$$
\widehat{\widetilde{\mathbf{F}}}(s)=\left[\widehat{\widetilde{f}}_{\lambda, \mu}(s)\right]=\frac{\left[p_{\lambda, \mu}(s)\right]}{q(s)},
$$

in the sense of minimizing the quadratic error $\varepsilon=\sum_{\lambda, \mu} \varepsilon_{\lambda, \mu}$ where $\varepsilon_{\lambda, \mu}=\left\langle e_{\lambda, \mu}, e_{\lambda, \mu}\right\rangle$. The solution is given by solving

$$
\left(\sum_{\lambda, \mu} \Psi_{\lambda, \mu}\right) \vec{a}=-\left(\sum_{\lambda, \mu} \vec{b}_{\lambda, \mu}\right),
$$

where the Gram matrices $\Psi_{\lambda, \mu}$ and the vectors $\vec{b}_{\lambda, \mu}$ are computed for each transfer function $\widehat{f}_{\lambda, \mu}(s)$ with the same Laguerre parameters $\alpha$ and $\gamma$.

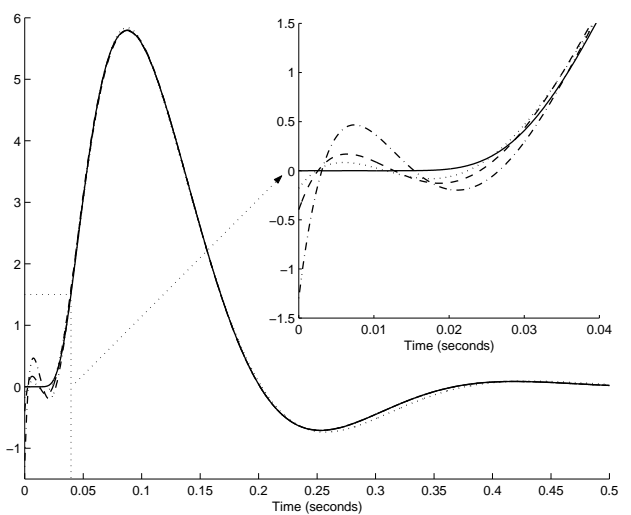

Fig. 1. Impulse responses of the original system, of its 4th-order model for $\left(\alpha_{1} ; \gamma_{1}\right)=(18.5 ; 37.0)$ (dashed line), for $\left(\alpha_{2} ; \gamma_{2}\right)=(8.95 ; 47.9)(\operatorname{dot}$ line) and of its 4th-order Padé model (dashed-dot line)

Starting with the Laguerre spectrum of each function $\hat{f}_{\lambda, \mu}(s)$ computed for common values $\alpha$ and $\gamma$ of the Laguerre parameters, the procedure of MIMO system reduction then follows:

1. Compute the Gram matrices $\Psi_{\lambda, \mu}$ and the vectors $\vec{b}_{\lambda, \mu}$ relative to the transfer functions $\widehat{f}_{\lambda, \mu}(s)$.

2. Form the Gram matrix $\Psi=\sum_{\lambda, \mu} \Psi_{\lambda, \mu}$ and the vector $\vec{b}=$ $\sum_{\lambda, \mu} \vec{b}_{\lambda, \mu}$ then solve $\Psi \vec{a}=-\vec{b}$ for $\vec{a}=\left[\begin{array}{llll}a_{0} & a_{1} & \ldots & a_{r-1}\end{array}\right]^{T}$.

3. Construct the reduced model $\widehat{\widetilde{\mathbf{F}}}(s)$ given in (20) by using (19) for all the values of $\lambda$ and $\mu$.

Note that the numerators $p_{\lambda, \mu}(s)$ could be optimized by solving

$$
\widehat{\widetilde{f}}_{\lambda, \mu}\left(\nu_{i}^{*}+\gamma-2 \alpha\right)=\hat{f}_{\lambda, \mu}\left(\nu_{i}^{*}+\gamma-2 \alpha\right)
$$

for $i=1,2, \ldots, r$ where $\nu_{i}^{*}$ stands for the negatives of the complex conjugate of the zeros of $q(s)$.

\section{NUMERICAL EXAMPLE}

To illustrate the utility of the method we present a typical application of model reduction of an infinite-dimensional system. The system considered is a distributed RC circuit whose irrational transfer function is [15], [16]

$$
\hat{g}(s)=\frac{1}{1+\frac{\sinh (\sqrt{R C s})}{\sqrt{R C s}}} \quad \text { with } R C=1 .
$$

From $\hat{g}(s)$, the first $N=150$ coefficients of a Laguerre model are recursively computed using [17] for two different weight functions $\varpi_{1}(t)=1$ and $\varpi_{2}(t)=e^{-30 t}$. The corresponding Laguerre parameters $\left(\alpha_{1} ; \gamma_{1}\right)=(18.5 ; 37.0)$ and $\left(\alpha_{2} ; \gamma_{2}\right)=(8.95 ; 47.9)$ used for the computation of the truncated Laguerre spectrum of $\hat{g}(s)$ came from the optimization method described in [16], [18]. Using the technique presented in this paper the following 4th-order reduced models are derived as

$$
\begin{aligned}
& \widehat{\widetilde{g}}_{1}(s)=\frac{-0.397 s^{3}+155.4 s^{2}-25589 s+1746201}{s^{4}+157.5 s^{3}+10611 s^{2}+239771 s+3493517}, \\
& \widehat{\widetilde{g}}_{2}(s)=\frac{-0.177 s^{3}+83.50 s^{2}-15866 s+1209479}{s^{4}+113.8 s^{3}+7820 s^{2}+166458 s+2443337},
\end{aligned}
$$

and are compared with the Padé approximation

$$
\widehat{\widetilde{g}}_{\text {Padé }}(s)=\frac{-1.298 s^{3}+357.2 s^{2}-46275 s+2693055}{s^{4}+205.6 s^{3}+15444 s^{2}+356292 s+5386110} .
$$

The impulse response of the original transfer function and those of the 4th-order models and Padé approximation are presented Fig. 1 and show the great accuracy of the presented model-reduction procedure. The time zoom near $t=0$ underlines the role played 
by the weight function $\varpi(t)=e^{-(\gamma-2 \alpha) t}$ : a choice of $\gamma>2 \alpha$, as used to derive $\widehat{\widetilde{g}}_{2}(s)$, produces an improvement of the reduced model quality around $t=0$ (to a comparative detriment of the quality for $t$ greater). If we define the relative weighted quadratic error by $Q \triangleq\langle g-\tilde{g}, g-\tilde{g}\rangle /\langle g, g\rangle$ we obtain $Q_{1}=2.18 \cdot 10^{-4}$ and $Q_{2}=6.10 \cdot 10^{-4}$ for the Laguerre-Gram reduced models, and $Q_{\text {Padé }}=1.32 \cdot 10^{-3}$ (computed for the weighting function $\varpi(t)=1$ ) for the Padé model. These results confirm the great accuracy of the model reduction procedure.

\section{CONCLUSION}

An efficient procedure for model reduction of finite or infinite dimensional transfer function described by a Laguerre expansion has been presented. Based on a one-order operator in the Laplace domain is constructed a set of basis functions from which is applied a procedure of quadratic error minimization. The use of a weight function in the inner product definition permits a control of the timeerror spreading of the reduced model. Integrals defining the required inner products are replaced by sums (finite in practice) expressed using the Laguerre spectrum of the original transfer function and can be computed recursively, allowing a numerically convenient calculation. We have shown that the reduced model preserves the first derivatives of the transfer function at a chosen point $s=\gamma-\alpha$ and has an impulse response that belongs to $L_{\varpi}^{2}\left(\mathbb{R}^{+}\right)$. The procedure has also been extended to MIMO systems. Presented work takes part inter alia of model reduction of infinite dimensional systems. An illustrative example has shown the efficiency of the procedure.

\section{ACKNOWLEDGMENT}

The authors wish to acknowledge the Brittany Region for financial support. They also would like to thank the reviewers for their valuable comments and suggestions.

\section{REFERENCES}

[1] P. R. Clement, "Laguerre functions in signal analysis and parameter identification," Journal of the Franklin Institute, vol. 313, no. 2, pp. 85-95, 1982.

[2] B. Wahlberg, "System identification using Laguerre models," IEEE Trans. Automat. Contr., vol. 36, no. 5, pp. 551-562, 1991.

[3] C. T. Chou, M. Verhaegen, and R. Johansson, "Continuous-time identification of SISO systems using Laguerre functions," IEEE Trans. Signal Processing, vol. 47, no. 2, pp. 349-362, 1999.

[4] P. M. Mäkilä, "Approximation of stable systems by Laguerre filters," Automatica, vol. 26, no. 2, pp. 333-345, 1990.

[5] - "Laguerre series approximation of infinite dimensional systems," Automatica, vol. 26, no. 6, pp. 985-995, 1990.

[6] G. A. Dumont, C. C. Zervos, and G. Pagean, "Laguerre-based adaptive control of $\mathrm{pH}$ in an industrial bleach plant extraction stage," Automatica, vol. 26, no. 4, pp. 781-787, 1990.

[7] L. Knockaert and D. D. Zutter, "Laguerre-svd reduced-order modeling," IEEE Trans. Microwave Theory Tech., vol. 48, no. 9, pp. 1469-1475, 2000.

[8] — , "Stable Laguerre-svd reduced-order modeling," IEEE Trans. Circuits Syst. I, vol. 50, no. 4, pp. 576-579, 2003.

[9] Y. Chen, V. Balakrishnan, C. K. Koh, and K. Roy, "Model reduction in the time-domain using Laguerre polynomials and Krylov methods," in DATE'2002, Paris, France, Mar. 4-8, 2002, pp. 931-935.

[10] R. Malti, D. Maquin, and J. Ragot, "Some results on the convergence of transfer function expansion on Laguerre series," in European Control Conference, ECC'99, Karlsruhe, Germany, 31 Aug.-4 Sep. 1999.

[11] L. C. Calvez, Contribution à l'étude des propriétés de la transformation $Z$ et de la transformation de Laguerre. Applications à l'analyse des signaux et circuits. Univ. de Brest, Brest, France: Thèse de Doctorat d'Etat, 1973.

[12] F. Tricomi, "Transformazione di Laplace e polinami di Laguerre," $R$. $C$. Accad. Nat. dei Lincei, vol. 21, pp. 232-239, 1935.

[13] W. T. Weeks, "Numerical inversion of Laplace transforms using Laguerre functions," J. ACM, vol. 13, pp. 419-426, 1966.
[14] H. Nagaoka, "Mullis-Roberts-type approximation for continuous-time linear systems," Electronics and Communications in Japan, vol. 70, no. 10, p. part 1, 1987.

[15] S. P. Johnson and L. P. Huelsman, "A high-q distributed-lumped-active network configuration with zero real-part pole sensitivity," Proc. IEEE, vol. 58, no. 3, pp. 491-492, 1970.

[16] R. Morvan, Modélisation de circuits et systèmes de dimension infinie. Univ. de Brest, Brest, France: Thèse de Doctorat, 2000.

[17] R. Morvan, N. Tanguy, P. Vilbé, and L. C. Calvez, "Simplified algorithm for Laguerre approximation of URC networks," Electronics Letters, vol. 35 , no. 16, pp. 1299-1300, Aug. 1999.

[18] N. Tanguy, P. Vilbé, and L. C. Calvez, "Optimum choice of free parameter in orthonormal approximations," IEEE Trans. Automat. Contr. vol. 40, no. 10, pp. 1811-1813, Oct. 1995. 